\title{
Prospect for Using Low-Element Adaptive Antenna Systems for Radio Monitoring Stations
}

\author{
Olga Shcherbyna \\ National Aviation University/Department of Electronics, Robotics and Monitoring and IoT Technologies/Kyiv, Ukraine, \\ 03058 \\ E-mail: shcherbyna_ol@nau.edu.ua
}

Maksym Zaliskyi

National Aviation University/Department of Telecommunication and Radioelectronic Systems/Kyiv, Ukraine, 03058

E-mail: maximus2812@ukr.net

\author{
Olena Kozhokhina \\ National Aviation University/Department of Avionics/Kyiv, Ukraine, 03058 \\ E-mail: kozhokhina@gmail.com
}

\section{Felix Yanovsky}

National Aviation University/Department of Electronics, Robotics and Monitoring and IoT Technologies /Kyiv, Ukraine, 03058

E-mail: yanovsky@nau.edu.ua

Received: 13 June 2021; Accepted: 21 August 2021; Published: 08 October 2021

\begin{abstract}
This article is devoted to the analysis of prospect to apply multifunctional adaptive antenna systems for radio monitoring stations. The review of publications done demonstrates that current antennas that are developed and used in radio monitoring systems to control and measure the parameters of electromagnetic radiation should be applicable to conduct accurate measurements in wide frequency range under the condition of interferences. The analysis shows that modern adaptive antenna systems are mostly developed for radar and telecommunications applications. In this context we consider possible ways to solve the problem of adapting radio monitoring devices to a complex electromagnetic environment using antenna systems with primary processing of received signals. It was found that the developers of the antennas, which are based on adaptive interference suppression methods, focus basically on the development and implementation of adaptation processes, limiting themselves only to solving electromagnetic compatibility problems. In such approach, the functions of direction finding and measurement of radiation field parameters important exactly for radio monitoring systems are mostly ignored. Therefore, this research area opens up a wide field for identifying new possibilities for constructing multifunctional antenna systems. Focusing on this direction of research, we consider as an example the constraction of a simple two-element adaptive antenna system, which can be used to measure the parameters of the electromagnetic field in radio monitoring systems. The main relations for the error of determining the direction of arrival of the interference signal with a simple two-element antenna are investigated. The influence of the stability of the antenna array parameters and functional units of signal processing onto the errors is estimated.
\end{abstract}

Index Terms: Adaptive Antenna Array (AAA), Antenna Array (AA), Electromagnetic Compatibility (EMC), Radio Monitoring Systems, Field Intensity, Polarization Parameters, Wave Arrival Direction.

\section{Introduction}

In the modern world, the number of telecommunications and other radio electronic devices is increasing permanently. This leads to stricter requirements for the rational use of the radio frequency resource in each country and globaly and ensuring of electromagnetic compatibility (EMC) from telecom operators and departments for the supervision of telecommunications and radio-electronic systems. On the other hand, when planning the use of the radio frequency resource, carefully selected frequencies are exposed to radio interference in practice, since theoretical calculations cannot always fully assess the electromagnetic environment in a particular place.These conditions lead to the need of additional measures for radio frequency control.

A significant role in these activities is assigned to over-the-air radio frequency control, which is an important tool for making decisions on the allocation of a radio frequency range for new radio facilities, increasing the efficiency of 
using the radio frequency resource (RFR) and ensuring conditions for the normal operation of licensed devices.Antenna or antenna system is one of the most important components of radio monitoring means.

In the case of a complex electromagnetic environment, antenna radio monitoring systems should not only make it possible to measure the parameters of the investigated electromagnetic field with high accuracy, but also adapt to the electromagnetic environment, allocating the analysed signal and suppressing interference signals. Many researchers pay attention to adaptive signal processing and, in particular, to the construction of adaptive antenna systems. This is because in the case of adaptive processing, the possibilities of suppressing interference signals (both unintentional and intentional) are significantly increased, including in the case of minimal a priori information about their parameters.

For example, in communication systems of the third, fourth and fifth generations ( $3 \mathrm{G}, 4 \mathrm{G}, 5 \mathrm{G})$, it is possible to use adaptive antennas. This allows to reduce the multipath effect, reduce the radiation power of base stations, reduce the influence of intersystem and intrasystem interference that is present in the frequency band of the useful signal, solve location problems, increase channel capacity, and others. The traditional means of signal processing include adaptive signal diversity due to the MIMO technology [1]. The military departments are showing great interest in adaptive antenna systems with signal processing since one of the most important tasks of military systems is stable operation under conditions of intentional interference.

However, for radio monitoring stations, simple antennas are usually used that require stability of parameters and functioning in a certain frequency band. Therefore, radio monitoring terminals need to use several antennas with a different functional purpose for one frequency band. This does not cause significant difficulties in the case of placement of antenna complexes on fixed terminals. But the antenna systems of mobile radio monitoring terminals cannot meet the current requirements for qualitative measurement of the basic parameters and coordinates of radiation sources. That is, new approaches to creating antenna systems are needed that would ensure the performance of several radio monitoring functions with a slight complication of the design of the primary signal processing circuit. To do this, it is possible to use known methods of adaptive signal processing, the development of which researchers have recently paid considerable attention. Based on the above, this article focuses on the prospects of using antenna systems with the functions of adaptation to complex electromagnetic conditions in radio monitoring stations.

\section{Literature Review and Problem Statement}

The topic of adaptive antenna systems has been widely studied since the 1950s [2-14]. The main function of any adaptive receiving antenna system for radar $[15,16]$ or communications [4-6] applications is to minimize the received power from one or more interference sources that are located within the coverage area of the antenna system. In the case of radar systems, the adaptive antenna can serve to suppress radar noise [16], as well as intentional or unintentional interference.In addition, the adaptive antenna system should have functions that allow the system to detect radio targets or receive signals from communication users with a specified signal-to-noise ratio. The ability of an adaptive antenna system to perform these functions is controlled by manyfactors, some of which include the number of adaptive antenna array elements, antenna aperture diameter, number and bandwidth of signal suppression, power and locations of interferers, and others.An adaptive antenna can be, for example, a micro- or millimetre-wave phased antenna array (AA) [17-21], which consists of several antenna elements. In this case, the main beam is electronically controlled in the review sector. Besides, an adaptive antenna can bea multi-beam $[22,23]$ and consist of several horn antennas that irradiate a parabolic reflector system or a lens antenna system [24]. The work [25] presents the design and analysis of smart antenna by circular pin-fed linearly polarized patch antenna. Patch antenna is a very prominent antenna in the microwave frequency spectrum due to its simplicity and compatibility with the printed circuit board technology. One example of a patch element that can be used as an antenna array element is presented in the article [26].

Adaptive antenna arrays (AAA) have found a wide field of application and have become an integral part of wireless communication systems [27-30]. There are different approaches to the implementation of the principles of increasing noise immunity by the terms of use, purpose and requirements for the radio system. The use of AAA for mobile and specialized communication networks of the microwave range with a high data rate is especially important due to the limited initial power of monolithic microwave integrated circuits [31,32]. Millimetre wavelength allows the use of massive AA in a transceiver to provide beamforming and spatial multiplexing.

Fully digital AAA is a design in which each antenna element is connected to a dedicated RF circuit [33-37]. It fully complies with classical MIMO technologies and thus results in improved performance in terms of data transfer rates. However, the implementation of fully digital AAA involves prohibitive costs, especially for multi-element arrays. This is because one dedicated RF channel is required per antenna element to implement fully digital beamforming. This is impractical in terms of both cost and energy consumption, especially at millimetre-wave frequencies [38].

Another problem of a full digital beamformer is the excessive need for real-time signal processing for antennas with high gain. For example, more than 1000 antenna elements may be needed to achieve an antenna gain of $30 \mathrm{~dB}$. This makes most beamforming algorithms impractical for commercial applications. Also, to perform wideband digital beamforming, each signal from / to the AA element is usually divided into some narrowband signals and processed separately, which also significantly increases the cost of digital signal processing. Therefore, a completely digital implementation of a multi-element broadband antenna array, especially at frequencies of the millimetre wavelength 
range, is almost unrealistic [39].

An alternative to fully digital AAA is hybrid designs that can be used to reduce associated costs. In these cases, the analoguebeamformers (phase shifters) are used to connect the RF circuits to the AA elements and control the directional characteristics of the array.The article [40] presents a hybrid receiving AAA (Fig. 1). In this antenna array, a large number of antenna elements are grouped into analogue subarrays, each of which uses an analoguebeamformer, and all subarray signals are combined using a digital beamformer to obtain the desired pattern [41].

Each element in the subarray (Fig. 1 [40]) has its own RF channel and uses an analogue device to phase shift in the intermediate frequency stage. The signals from the subarray elements are combined after the analogue phase shifters and the total beamforming signal is transferred to the intermediate frequency range and then converted to digital form. Thus, the complexity of the digital beamformer is reduced by a factor equal to the number of elements in the subarray. For example, for a hybrid AA with 1024 elements and 64 subarrays, each of which includes 16 elements, only 64 inputs to the digital beamformer are needed, and the complexity is reduced to one-sixteenth. The cost of digital equipment is also reduced.The digital beamformer evaluates the angle of arrival information to control the phases of the phase shifters in the analogue subarrays and also adjusts the digital weights of the subarray output signals to form the desired beam.

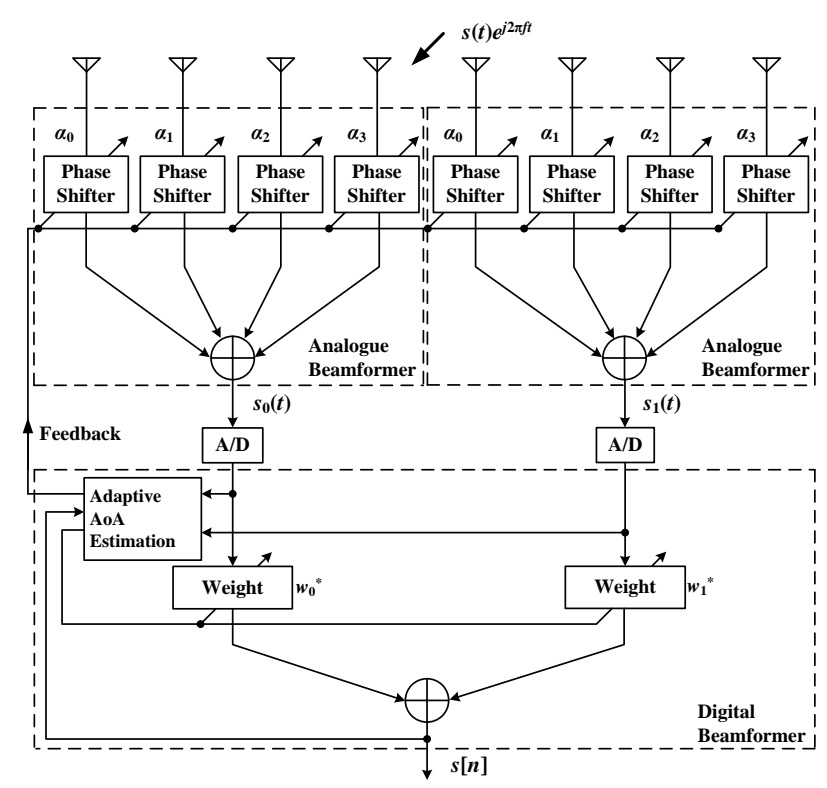

Fig.1. Hybrid block diagram of beam formation with two linear subarrays [40]

It should be noted that the subarray technology has been used over the past decades [29, 41-44]. But the main idea was to use a time delay device for each phased antenna subarray to expand the bandwidth and eliminate analogue phase shifters for applications that only require a limited viewing angle. This condition is not suitable for radio monitoring systems, especially in the case of mobile systems. Knowledge of the angle of arrival and polarization of the wave is of great importance for the reception quality. The paper [45] investigated the estimates of these parameters for an information signal in millimetre-wave systems using hybrid AAA with double polarization dipoles as elements. Antennas with controllable polarization are widly used for different applications where analysis of polarization structure of the signal is necessary $[46,47]$.

Following the research of signal processing methods for overall AA, it is recommended to use antenna elements with omnidirectional radiation [48-50]. Each AA element has two orthogonal dipoles [51], which are located along the $\mathrm{x}$ and $\mathrm{y}$-axes, respectively. Dipoles are used to measure the components of the electric field intensity that are projected onto the $\mathrm{x}$ and $\mathrm{y}$ directions.

The incident wave can simultaneously excite two signals in the antenna from orthogonal dipoles. Accordingly, two sets of signals from dipoles along the $\mathrm{x}$-axis and dipoles along the $\mathrm{y}$-axis can be generated in the AA. In terms of signal reception, they represent two copies of the signal that can be sequentially combined to enhance the signal-to-noise ratio at the decoder. The signal diversity depends on the signal-to-noise ratio of each dipole. This requires an estimate of the arrival angle and the polarization of the incident wave.Wave reception with the use of four local AAs with double polarization and a simplified block diagram of the primary signal processing is illustrated in Fig. 2 [45]. A similar detailed description of adaptive antenna systems for solving radio monitoring problems has not been found in the literature. The examples of structural diagrams of AAA, which were considered above [40, 45], can be used in telecommunication systems for various purposes of the microwave ranges to increase the reliability of communication. But the use of such AAAs as antenna systems for radio monitoring is significantly limited due to some disadvantages. 


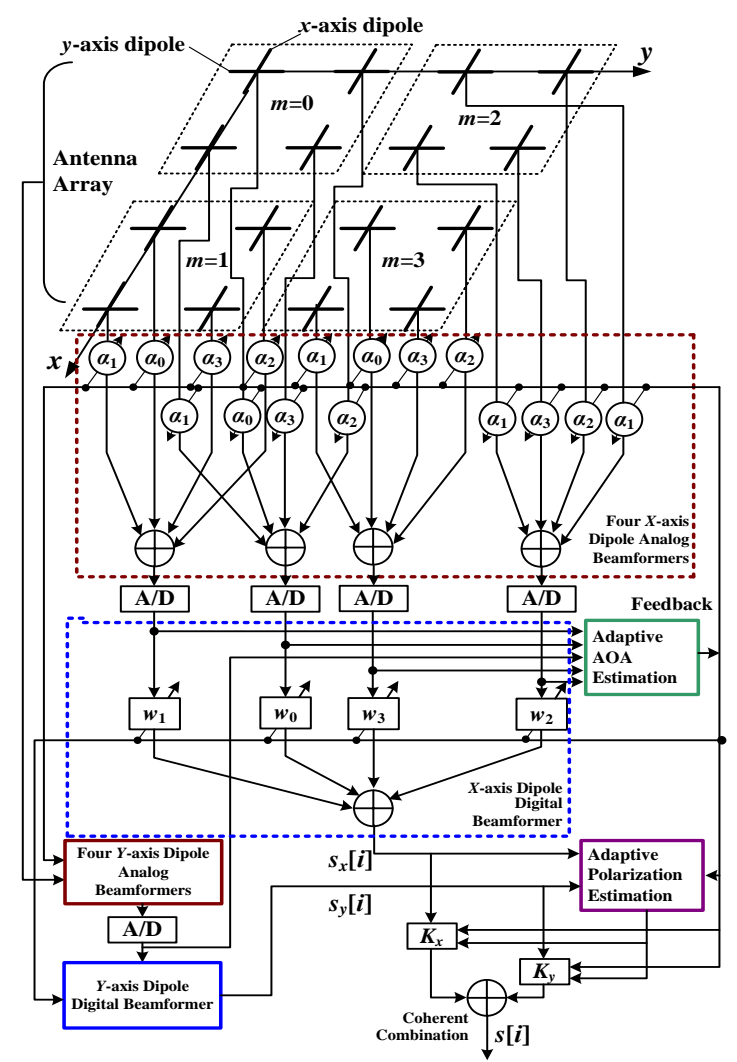

Fig.2. Block diagram of an adaptive antenna system with theAA, which consists of four subarrays [42]

First of all, the proposed adaptive systems for telecommunication applications have AAs with a sufficiently large number of elements. This is the main limitation in radio monitoring systems, especially mobile ones. Since the control over radiation is carried out not only in the centimetre and millimetre ranges but also at longer wavelengths, the use of a multi-element antenna array can significantly increase the dimensions of the antenna unit of the system.

Since in the considered AAs, for adaptation to a complex electromagnetic environment and to eliminate the influence of interference, electrical beamforming is used, the sector of the space for review, and, consequently, for measuring the parameters of the electro electromagnetic field, is limited to a hemisphere. In addition, the field of view narrows even more due to errors that arise when the pattern is deformed as a result of wave interference at the aperture edges of the AA.

One of the most important requirements for a modern mobile radio monitoring system is the review of the entire space in the azimuthal plane and the automatic determination of the direction of arrival of the measured signal. Therefore, the first way to solve this problem is to use an omnidirectional antenna in the azimuthal plane. But to accurately measure a wide range of electromagnetic field parameters, it is necessary to use several antennas that are spaced apart. The second way is to use antenna arrays with mechanical rotation to scan space and align the direction of maximum reception with the direction of arrival of the wave, which is necessary for accurate signal measurement.

The third of the noted disadvantages of using AAA of telecommunication systems for radio monitoring systems is the complexity of the primary signal processing scheme, which in turn can lead to additional errors in measuring the required parameters of the radiation field. Even in the AAA circuits discussed in this article above, the developers have tried to simplify signal processing circuits by using a combination of analogue and digital circuit elements. That is, there is a need to simplify the block diagram of the primary signal processing.

But, despite the great limitations, some of the principles of AAA design for telecommunication systems can be used for the construction of radio monitoring antenna systems. One of these principles is the use of orthogonal dipoles (dipole antennas) as elements of AA of radio monitoring stations. This will allow separating the received wave in a linear orthogonal polarization basis and, therefore, with sufficiently high accuracy to measure the polarization parameters of the received wave. It is only necessary to develop a mathematical apparatus for this task and build a structural diagram for the implementation of these mathematical foundations.

Also, in some cases, for radio monitoring systems, it is possible to use the principles of AAA construction using phase shifters in the structural diagram to control the position of the radiation pattern. But, as noted above, this is only suitable when the limited review sector is sufficient to control the field parameters. For example, in cases where the sector where the source of the measured signal is located is known in advance and the signal source does not go beyond its limits. But in the case of mobile radio monitoring systems, this condition is contradictory. 


\section{Antennas for Radio Monitoring Systems}

In practice, all radio monitoring tasks are solved in a complex electromagnetic environment, especially when it comes to modern megacities. Therefore, this requires the use of a wide range of technical means, including a large number of various antennas and antenna systems. All radio monitoring antennas, according to their functional purpose, can be conditionally divided into three main groups: control antennas, measuring antennas, direction-finding antennas. The main difference between antennas for radio monitoring and antennas used in other systems, such as telecommunications, is that they work only in the receive mode. For high-quality monitoring of the electromagnetic environment, the radio monitoring station must have a certain set of antennas. In the general case, the set of stationary antennas shortwave station includes at least such antennas: direction-finding antenna;frame direction finder for the frequency band 2-3 MHz;an omnidirectional antenna for the frequency band 2-30 MHz;a high-gain antenna for receiving horizontally polarized waves; a high-gain antenna for receiving vertically polarized waves.

The VHF/UHF antenna set of the monitoring station can include up to ten different antennas for different frequency bands. One of the variants of the block diagram of a multichannel radio monitoring station for frequencies of $9 \mathrm{kHz}-3 \mathrm{GHz}$ is shown in Fig. 3 [52]. As can be seen from the diagram, the station should include a set of antennas, which include antennas for detecting, direction finding and measuring the investigated electromagnetic wave. Also, this set should provide coverage of the entire frequency range necessary for research. Stringent requirements are imposed on the parameters of the measuring antennas since in the future the measurement results are used as the basis for checking and making a decision on the compliance of the radio-electronic system with the conditions of permits for operation.

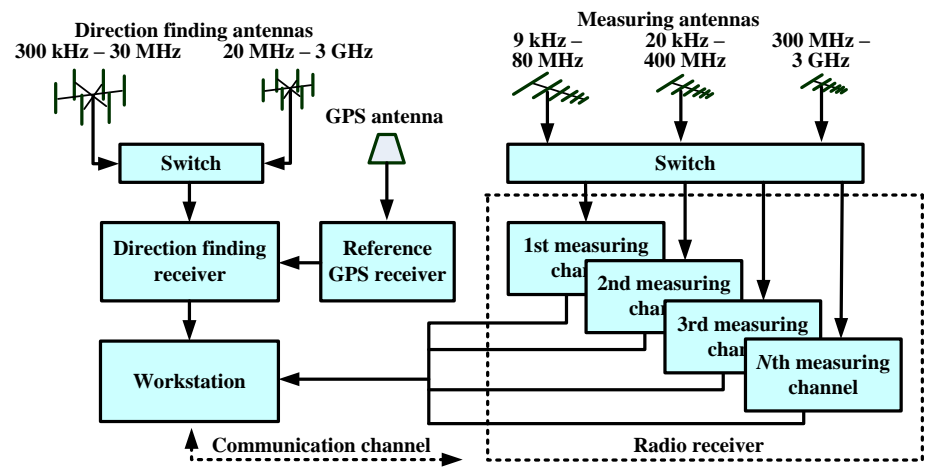

Fig.3. Block diagram of a multichannel radio monitoring station [52]

An example of a combination of omnidirectional and directional broadband antennas for radio monitoring is shown in Fig. 4 [53]. The antennas are installed on one platform, the spatial position of which is adjustable. Active and passive omnidirectional antennas are used in the frequency range of $10 \mathrm{kHz}-1600 \mathrm{MHz}$. Log-periodic antennas cover the range of 80-1300 MHz. Reflector antennas cover the range above $1 \mathrm{GHz}$. The different antennas are selected by a remote RF switch, the signal from which is transmitted through the rotator using a rotating joint.

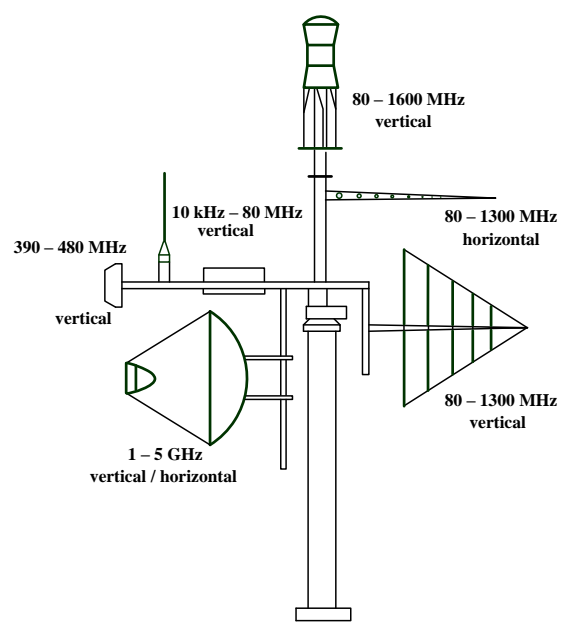

Fig.4. Combination of omnidirectional and directional antennas for radio monitoring [53]

There are many works devoted to the development of antennas that can be used in radio monitoring systems. The article [54] presents an ultra-wideband monopole antenna for EMC studies. The proposed measuring antenna can be 
used to detect the emissions of many digital devices, such as mobile phones and other mobile communication systems. In [55], two new types of ultra-wideband antennas for EMC measurements are presented. Both antennas are compact in size and can have multiple ports for flexibility in measurement tests. The pattern can be directional or multi-lobe. These antennas are inexpensive to manufacture, light in weight and easy to install, which is especially important for lowfrequency antennas. Article [56] presents a developed conical horn antenna that has such advantages as broadband, simple power circuit, low profile compact dimension with good performance. The paper [57] presents a new biconical broadband antenna, which is used for EMC test applications. Compared to a conventional biconical antenna, the proposed antenna has a small dimension and good electrical performance. The work [58] is devoted to the development of an algorithm for calculating the design of a printed log-periodic antenna, which can be used as a measuring antenna for mobile radio monitoring systems. Mathematical modelling and experimental study of three log-periodic antennas, for the manufacture of which various substrate materials are used, have been carried out.

As can be seen from the structural diagrams and examples of monitoring antenna systems, one monitoring station must have a set of antennas of various designs. In many cases, these antennas are located in a limited area (especially for mobile monitoring stations), which degrades the EMC of the station elements. In addition, in practice, radio monitoring is carried out under the influence of many factors, such as industrial and atmospheric noises, radiation from radio transmitters, etc. This leads to the need to use additional means (technical or software) to isolate the required signal.

\section{Methods of Interference Suppression by Antenna Systems}

Methods for suppressing the influence of interference using antenna systems with primary signal processing can be conditionally divided into compensation and adaptive methods. The compensation principle in the general case can be demonstrated using the block diagram of a two-channel compensation antenna system (Fig. 5).

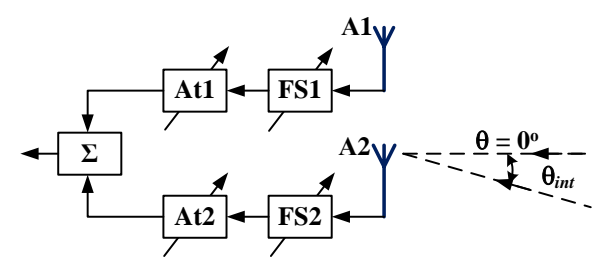

Fig.5. Block diagram of a two-channel compensation antenna

This antenna system uses two channels, which consist of antennas (A1 and A2), controlled phase shifters (FS1 and FS2) and controlled attenuators (At1 and At2). The outputs of the antenna channels are connected to the inputs of the adder $\Sigma$. Let's assume that the antenna system is tuned to the direction of arrival $\theta=0^{\circ}$ of the desired signal. The interference comes in a direction $\theta_{\text {int }}$. Interference levels in channels 1 and 2 are equalized using controllable attenuators At1 and At2. Phase shifters FS1 and FS2 create a phase shift equal to $\pi$ between the interference signals in the two channels. As a result, the interference level at the output of the adder $\Sigma$ will be equal to zero.

Antennas A1 and A2 have different directional characteristics, which together can change the directional properties of the antenna system $\mathrm{A} \Sigma$ as a whole. Let the main antenna $\mathrm{A} 1$ of the system have a greater directivity than the antenna A2 of the second (compensation) reception channel.

Based on the directional properties of the antennas, the compensation procedure can be explained as follows. Fig. 6 shows the radiation patterns (RP) of the main (curve 1), compensation (curve 2) and total (curve 3) antennas. To prevent a decrease in the level of the useful signal in channel 1, a signal amplifier can be inserted into channel 2.

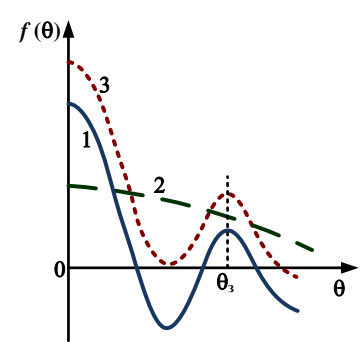

(a)

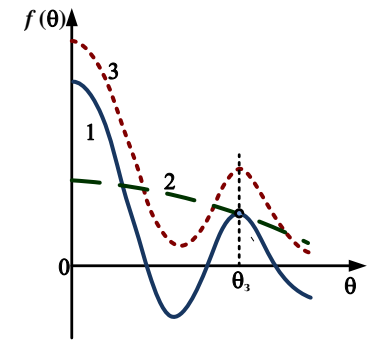

(b)

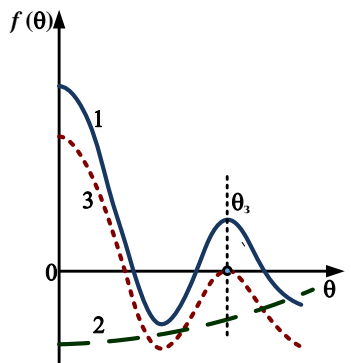

(c)

Fig.6. Directional patterns of antennas with amplitude and phase compensation: $a$ - in the absence of compensation; $b$ - when aligned with the help of controlled signal attenuators in the direction $\theta_{\text {int }}\left(\left|f_{1}\left(\theta_{\text {int }}\right)\right|=\left|f_{2}\left(\theta_{\text {int }}\right)\right|\right) ; c$ - after an additional phase shift $\pi$ using controlled phase shifters $\left(f_{1}\left(\theta_{\text {int }}\right)=-f_{2}\left(\theta_{\text {int }}\right), f_{\Sigma}\left(\theta_{\text {int }}\right)=0\right)$. 
The considered compensation method provides for simultaneous control of both the amplitude and phase of the signal in the receiving channels. But there are cases when it is advisable to use control of only one of the signal parameters. According to the principle of operation, compensation methods can be divided into amplitude, phase and amplitude-phase. To compensate for interference by any of these methods, it is necessary to use at least two signal reception channels of the antenna system.

An adaptive antenna array is a type of antenna in which the parameters are changed adaptively according to the influence of external factors. Such factors are, as a rule, the influence of active and passive interference, in the presence of which the possibility of adaptation increases the quality of signal reception.

AAA is an antenna array and a signal receiving and processing device that automatically adjusts RP to improve the quality of the desired signal reception [59-62]. AAA makes it possible to ensure thehigh quality of the received signal in a complex electromagnetic environment, in a dynamic interference situation, when placing antenna systems on mobile terminals. As a quality criterion, the signal-to-noise ratio or the deviation of the selected parameter of the useful signal from the known standard is most often chosen.

By definition, an adaptive antenna has the following components: an antenna array of $N$ elements, the design and location of which depends on the purpose of the antenna and it is the most significant factor in limiting the capabilities of an adaptive antenna; a circuit for forming the RP, to the inputs of which the outputs of all $N$ elements of the antenna array are connected; the basis of this scheme is a four-port network with variable transmission coefficients; an adaptive processor that calculates the value of the complex transmission coefficients of the four-pole networks of the circuit for forming the RP.

The adaptation principle is described in detail in many works [63-65]. By adjusting the complex weights of the AA elements, it is possible to completely suppress the interference signal. For a more visual representation of the adaptation principle, it is possible to demonstrate the change in the directional pattern by the change in the weights.

The normalized directivity characteristic of an $N$-element uniform linear AA, which consists of omnidirectional elements, can be described using the formula [63]

$$
G(\theta)=10 \lg \left(\frac{\left|\mathbf{w}^{T} \mathbf{a}(\theta)\right|^{2}}{\mathbf{w}^{H} \mathbf{w}}\right)=10 \lg \left(\left|\sum_{n=0}^{N-1} w_{n} \exp \left(-i \frac{2 \pi n d \sin \theta}{\lambda_{0}}\right)\right|^{2} / \mathbf{w}^{H} \mathbf{w}\right)
$$

where $\mathbf{w}$ is thematrix of weights; $\mathbf{a}(\theta)=\left[1, \exp \left(-i 2 \pi \sin \theta_{s} / \lambda_{0}\right), \ldots, \exp \left(-i(N-1) 2 \pi \sin \theta_{s} / \lambda_{0}\right)\right]^{T}$ is the spatial vector of AA elements.

Fig. 7, $a$ shows the normalized RP (1) for a two-element AA without introducing weights into the RP forming scheme. In Fig. 7,b the normalized RP for the same AA is presented with the introduction of weights $w_{1,1}=w_{1,2}=w_{2,1}=0.5$ and $w_{2,2}=-0.5$. It can be seen that in the second case, the zero of the RP is located exactly along the azimuth of $30^{\circ}$ which coincides with the direction of arrival of the interference signal.

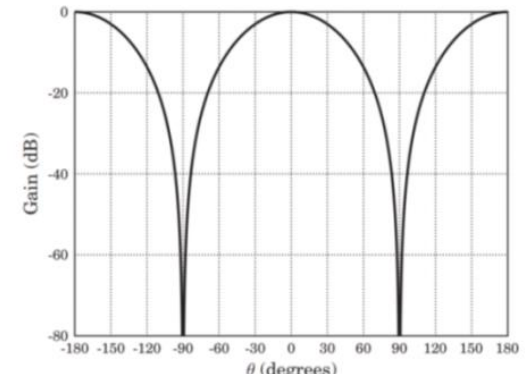

(a)

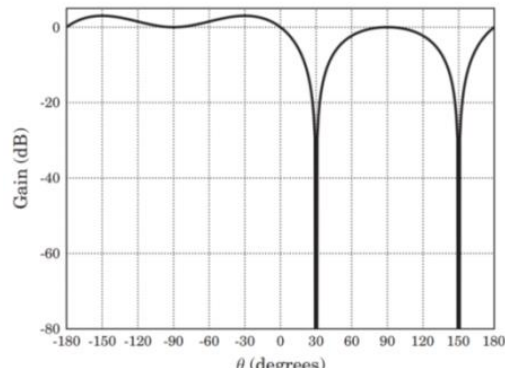

(b)

Fig.7. The normalized RP (1) for two-element AA: $a$ - without the introduction of weights; $b$ - with the introduction of weights [63]

This simple example shows how the interference signal $n(t)$ is completely "zeroed" by adjusting the complex weights of the AA elements. In this case, the output signal will be equal to the useful signal $s(t)$. This model can be easily extended for an N element AA. Such an array can restore the desired useful signal and completely suppress $(N-1)$ interference signals.

The main disadvantage of AAAs is the presence of a transient process, which is necessary to complete the adaptation.Therefore, turning on spaced-apart sources of interference for a time interval less than the adaptation period of the RP of AAA is a very effective method that deprives the corresponding radio engineering systems of noise immunity. 


\section{Two-element Adaptive Antenna System for Radio Monitoring Systems}

Let's consider one of the simplest examples of constructing an adaptive antenna for radio monitoring systems, which consists of two elements only. Unfortunately, in this case, the spatial selectivity of the antenna cannot be used directly. This is since the direction to the interference source is not known in advance and the interference source can move. Also, the dual-element AA has a fairly wide RP, which significantly reduces the spatial resolution. An increase in the number of elements to increase spatial selectivity leads to an increase in the costs of manufacturing and maintaining the antenna and may make it impossible to use it as an antenna for mobile radio monitoring stations.

That is, in a low-element antenna array, spatial selectivity must be provided by signal processing. The principle of operation of such a two-element antenna is described in monograph [66]. To build an antenna on this principle, it is necessary to solve two problems: automatic detection and determination of the spatial location of the interference source, automatic compensation of interference in the received signal.

The solution to the formulated problems can be implemented using the scheme shown in Fig. 8. Two radiators $\mathrm{A}_{1}$ and $\mathrm{A}_{2}$, non-directional in the horizontal plane, are located at a distance $d$ from each other so that the line connecting their phase centres is perpendicular to the direction of arrival of the wave with the useful signal (Fig. 9).

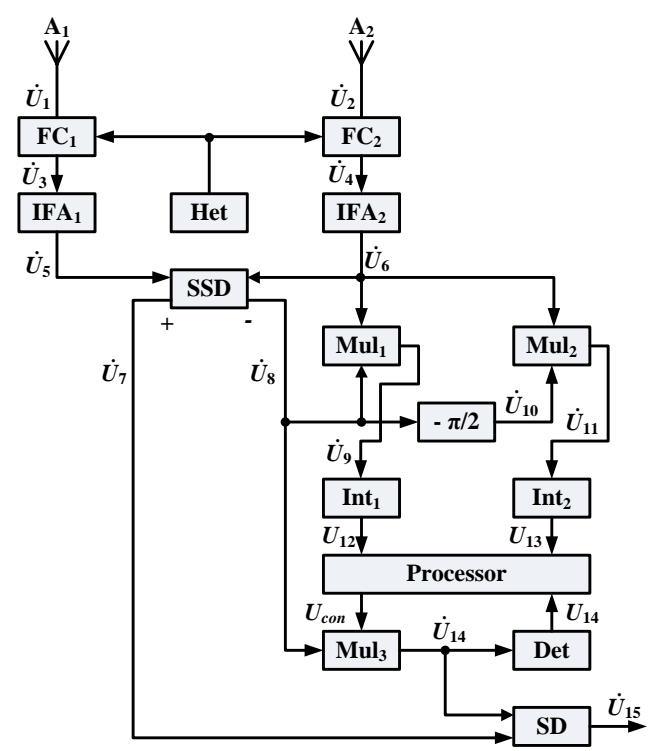

Fig.8. Block diagram of a two-element adaptive antenna array

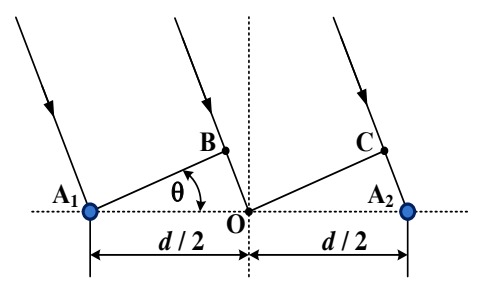

Fig.9. Schematic representation of the antenna array elements relative to the arrival direction of the electromagnetic wave

Let the direction of arrival of the interference wave deviate at an angle $\theta$ from the direction of the perpendicular to the arrangement line of the antenna array elements. Due to this, the interference voltages at the terminals of the array elements will be out of phase, while the voltages of the useful signal will be in phase:

$$
\left.\begin{array}{c}
\dot{U}_{1}=l_{\text {eff }} \dot{E}_{s}+l_{\text {eff }} \dot{E}_{i n t} e^{i \psi}=\dot{U}_{s}+\dot{U}_{\text {int }} e^{i \psi} ; \\
\dot{U}_{2}=l_{e f f} \dot{E}_{s}+l_{e f f} E_{i n t} e^{-i \psi}=\dot{U}_{s}+\dot{U}_{i n t} e^{-i \psi}
\end{array}\right\}
$$

where $l_{\text {eff }}$ is the effective lengths of the AA elements (must be equal to each other); $\dot{E}_{s}$ is signal field intensity; $\dot{E}_{\text {int }}$ is interference field intensity; $\psi=k d \sin \theta / 2$ is phase shift, which is due to the difference in the path of the beams OB and $\mathrm{CA}_{2}$ (Fig. 9); $\dot{U}_{s}, \dot{U}_{\text {int }}$ are complex amplitudes of signal and interference voltages at antenna terminals.

To ensure high accuracy of signal processing and the use of standard integrated circuits in the frequency 
processing device, the received electromagnetic waves are converted using a heterodyne Het and frequency converters $\mathrm{FC}_{1}$ and $\mathrm{FC}_{2}$. This allows the frequency-converted voltages to maintain phase and amplitude relations. The voltages at the outputs of $\mathrm{FC}_{1}$ and $\mathrm{FC}_{2}$ can be written as

$$
\dot{U}_{3}=K_{\mathrm{FC} 1} \dot{U}_{1} ; \quad \dot{U}_{4}=K_{\mathrm{FC} 2} \dot{U}_{2}
$$

where $K_{\mathrm{FC} 1}$ and $K_{\mathrm{FC} 2}$ are the transfer coefficients of the frequency converters $\mathrm{FC}_{1}$ and $\mathrm{FC}_{2}$.

The amplitudes of the converted signals increase, passing through the intermediate frequency amplifiers IFA $\mathrm{A}_{1}$ and $\mathrm{IFA}_{2}$. The signals from the outputs of the amplifiers have the form

$$
\left.\begin{array}{c}
\dot{U}_{5}=a_{1} K_{\mathrm{FC} 1} \dot{U}_{1}=a_{1} K_{\mathrm{FC} 1}\left(\dot{U}_{s}+\dot{U}_{i n t} e^{i \psi}\right) \\
\dot{U}_{6}=a_{2} K_{\mathrm{FC} 2} \dot{U}_{2}=a_{2} K_{\mathrm{FC} 2}\left(\dot{U}_{s}+\dot{U}_{i n t} e^{-i \psi}\right)
\end{array}\right\},
$$

where $a_{1}$ and $a_{2}$ are the gain factors.

The summation-subtraction device (SSD) generates signals, which, taking into account expressions (2), (3) and (4), are written in the form

$$
\begin{gathered}
\dot{U}_{7}=\dot{U}_{5}+\dot{U}_{6}=2 A\left(\dot{U}_{s}+\dot{U}_{i n t} \cos \psi\right), \\
\dot{U}_{8}=\dot{U}_{5}-\dot{U}_{6}=i 2 A \dot{U}_{i n t} \sin \psi .
\end{gathered}
$$

where $A=a_{1} K_{\mathrm{FC} 1} K_{\Sigma}=a_{2} K_{\mathrm{FC} 2} K_{\Delta} ; K_{\Sigma}$ is thetransmission coefficient of voltage summing channel; $K_{\Delta}$ is transmission coefficient of voltage subtraction channel.

At the same time, the signal $\dot{U}_{6}$ is fed to the multipliers $\mathrm{Mul}_{1}$ and $\mathrm{Mul}_{2}$. The other inputs of the multipliers are connected to voltages $\dot{U}_{8}$ and $\dot{U}_{10}$, which is a voltage $\dot{U}_{8}$ shifted by $90^{\circ}$ (using a phase shift element), that is

$$
\dot{U}_{10}=2 \dot{U}_{i n t} \sin \psi
$$

At the output of the first multiplier $\mathrm{Mul}_{1}$, a voltage $\dot{U}_{9}$ is obtained, which is determined in the time domain as follows

$$
U_{9}(t)=M_{1} U_{6}(t) U_{8}(t)=M\left[U_{s} U_{i n t} \cos \omega_{s} t \cos \omega_{i n t} t \sin \psi+U_{\text {int }}^{2} \sin \psi \sin \omega_{i n t} \cos \left(\omega_{i n t} t-\psi\right)\right]
$$

where $M=K_{\mathrm{Mul}} a_{2}^{2} K_{\mathrm{FC} 2}^{2} K_{\Delta} ; K_{\mathrm{Mul}}$ is transmission coefficient of the multipliers $\mathrm{Mul}_{1}$ and $\mathrm{Mul}_{2} ; \omega_{s}$ and $\omega_{\text {int }}$ are circuit frequencies of signal voltage and interference.

At the output of the multiplier $\mathrm{Mul}_{2}$, taking into account (4) and (6), the voltage is obtained

$$
\left.U_{11}(t)=M_{2} U_{6}(t) U_{10}(t)=M \mid U_{s} U_{i n t} \cos \omega_{s} t \cos \omega_{i n t} t \sin \psi+U_{i n t}^{2} \sin \psi \cos \omega_{i n t} \cos \left(\omega_{i n t} t-\psi\right)\right]
$$

After integrating the signals $U_{9}(t)$ and $U_{11}(t)$ over time for the period $T$, the average values of the voltages at the outputs of the integrators $\mathrm{Int}_{1}$ and $\mathrm{Int}_{2}$ are found

$$
\left.\begin{array}{c}
U_{12}=K_{i} \int_{0}^{T} U_{9}(t) d t \\
U_{13}=K_{i} \int_{0}^{T} U_{11}(t) d t
\end{array}\right\}
$$

where $K_{i}$ is thetransmission coefficient of the integrators.

Since the signal and interference voltages are incoherent $\left(\omega_{s} \neq \omega_{i n t}\right)$, then after integrating the voltages $U_{12}$ and $U_{13}$ will be constant and equal to

$$
\left.\begin{array}{c}
U_{12}=K_{i} M U_{\text {int }}^{2} \sin ^{2} \psi \\
U_{13}=K_{i} M U_{i n t}^{2} \sin \psi \cos \psi
\end{array}\right\} .
$$


The processor calculates the value ctg $\psi$ from the voltage values (8) $\operatorname{ctg} \psi=U_{13} / U_{12}$ and the control voltage is forme

$$
U_{c o n}=b c \operatorname{tg} \psi=b U_{13} / U_{12} .
$$

The control voltage is connected to one of the inputs of the third multiplier $\mathrm{Mul}_{3}$, to the second input of which voltage $\dot{U}_{8}$ is applied. The voltage at the output of $\mathrm{Mul}_{3}$ will be equal

$$
\dot{U}_{14}=\dot{U}_{8} b c \operatorname{tg} \psi=b \dot{U}_{8} U_{13} / U_{12} .
$$

If the voltage $\dot{U}_{14}$ is detected by the detector (Det), then, as follows from expressions (5) and (10), the voltage amplitude is obtained

$$
U_{14}=2 A b U_{i n t} \cos \psi
$$

For the correct formation of the voltage $U_{14}$, its amplitude in the processor is compared with the reference voltage, which is calculated as

$$
U_{0}=\sqrt{U_{13}^{2} / U_{12}}=\sqrt{K_{i} M} U_{i n t} \cos \psi
$$

The voltage $\dot{U}_{14}(10)$ and the voltage $\dot{U}_{7}(5)$ are fed to the inputs of the subtraction device (SD), at the output of which the output signal is formed

$$
\dot{U}_{15}=\dot{U}_{7}-\dot{U}_{14}=2 A \dot{U}_{s} .
$$

From equality (12), the necessary transmission coefficients of the considered circuits are determined. The constant factor from expression (11) must satisfy the following equality

$$
\sqrt{K_{i} M}=2 A
$$

and the factor $b$ of expression (9) must be equal to one. If these requirements are met, the value of the control voltage (9) is calculated from the condition $\left|U_{0}-U_{14}\right| \leq \varepsilon$, where $\varepsilon$ is apermissible error, which is determined by the minimum allowable signal-to-noise ratio.

From expression (7) it can be seen that with the transfer coefficient of the SSD, which is equal to one, and the transfer coefficient of the multipliers $\mathrm{Mul}_{1}$ and $\mathrm{Mul}_{2} K_{\mathrm{Mul}}=1$, equality (13) will be satisfactory at $K_{i}=1$. At stable values of the transmission coefficients, the implementation of condition (13) does not encounter any difficulties. As a result, the voltage $\dot{U}_{15}$ at the output of the antenna device will be a useful signal accurate to the additive component $2 A \varepsilon \dot{U}_{\text {int }} \cos \psi$.

In contrast to traditional case, the considered example of a simple two-element antenna system allows to effectively suppress interference at the frequency of the useful signal if the radiation of the interference source comes at an angle to the direction of arrival of the signal. This antenna system allows to determine the direction of arrival of the signal based on suggested above mathematic tool, which can be easily implemented digitally. The processor calculates the meridional angle using the corresponding software. Compared to the antennas developed for adaptive telecommunication systems that were discussed in the review section, the presented low-element antenna system has many advantages. In particular, it has extended functionality and it is a simplified structure based on the antenna array consisting of two elements only. Of course, in the future the antenna array can be expanded to, for example, four elements for improving its accuracy and further expanding its functionality.

\section{Experimental Results and Discussion Section}

It is known [7] that the antenna, which uses the elimination of the interference signal in the output voltage with relatively simple processing, cannot work effectively at the small angular spacing between the signal and interference 
sources. Nevertheless, the simplicity of the signal processing device and the ability to obtain additional information about the location of the signal source make such an antenna array promising. The use of such antenna arrays for radio monitoring is possible, provided that the answers to two basic questions are found. The first is what is the minimum angular separation between signal and interference sources. The second one is what is the error in determining the direction to the interference source.

First of all, it is necessary to consider how the signal-to-noise ratio changes depending on the direction angle $\theta$ of the interference wave arrival [67]. Besides, the minimum value of the angle $\theta_{\min }$ should be determined based on the permissible changes in the control voltages in the device. That is important to estimate the angle $\theta_{\min }$ at the maximum allowable voltage values that are produced by the processor. And, finally, it is necessary to determine the dependence of the angle measurement accuracy on the stability of the antenna array parameters and functional units of the signal processing block diagram. The block diagram (Fig. 8) shows the voltages at the corresponding points of the signal processing system. The output voltage $\dot{U}_{15}$ carries information, which is necessary for monitoring, that is, the voltage $\dot{U}_{15}$ is the useful signal. Signals $\dot{U}_{1}$ and $\dot{U}_{2}$ contain a mixture of signal $\dot{U}_{s}$ and noise $\dot{U}_{\text {int }}$. The ratio of the amplitudes of the two signals is defined as $\gamma=U_{s} / U_{i n t}$.

From the operation principle of the device, it follows that the signal voltage at the output is

$$
\dot{U}_{\text {int }}=\left(\dot{U}_{7}-\dot{U}_{14}\right) / 2 A=\dot{U}_{15} / 2 A \text {, }
$$

where $A$ is some constant, which is determined by the transfer coefficients of the components of the signal processing circuit.

In the feedback circuits, the voltage $U_{14}$ is compared with the reference voltage $U_{0}$ and the control voltage $U_{\text {con }}$ is generated by the magnitude of the mismatch of these voltages. The control voltage changes the transmission coefficient of the multiplier unit $\mathrm{Mul}_{3}$. This voltage is defined as

$$
\dot{U}_{14}=2 A \dot{U}_{i n t} \cos \psi+|\varepsilon|=\dot{U}_{0}+|\varepsilon|,
$$

where $\varepsilon$ is a permissible error, which is determined by the minimum permissible signal-to-noise ratio.

Considering that $\dot{U}_{7}=2 A\left(\dot{U}_{s}+\dot{U}_{i n t} \cos \psi\right)$, the following signal-to-noise ratio is found at the output of the signal processing device $\gamma^{*} \leq\left|\dot{U}_{15} /\left(U_{0}-U_{14}\right)\right|=2 A U_{s} /|\varepsilon|$.

The processor can provide an inconsistent value of the order of 0.01 or less. Therefore, the signal-to-noise ratio at the output of the signal processing device increases by several orders.

To estimate the minimum separation angle of signal and interference sources, the relation between the control voltage $U_{c o n}$, which is generated in the processor, and the phase shift $\psi$ between the components of the interference signal is used. From the principle of operation of the antenna, it follows that $U_{c o n}=b \operatorname{ctg} \psi$, where $b$ is aconstant coefficient, which is measured in volts. Taking into account that the phase shift is determined by the difference in the path of the beams to the elements of the antenna array $(\psi=(k d \sin \theta) / 2)$, the following values are found

$$
\begin{gathered}
U_{c o n}=b \operatorname{ctg}((\pi d \sin \theta) / \lambda), \\
\theta=\arcsin \left[\left(\lambda \operatorname{arctg}\left(U_{c o n} / b\right)\right) / \pi d\right] .
\end{gathered}
$$

Expressions (14) and (15) make it possible to determine the angle $\theta_{\min }$. The minimum separation angle of signal and interference sources depends on the distance $d / \lambda$ between the elements of the antenna array and the maximum possible value of the ratio of the control voltage to the coefficient $b$. According to the formula (14), the graph of dependence is built (Fig. 10). 


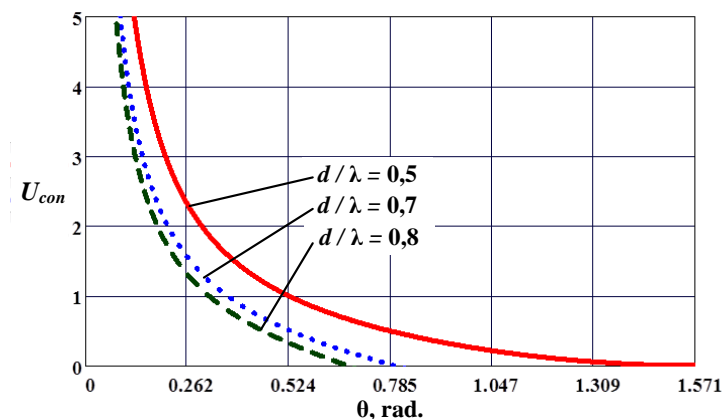

Fig.10. Dependence of the control voltage on the arrival angle of the interference electromagnetic wave at the fixed relative distance between the antenna array elements.

The graph in Fig. 10 makes it possible to determine without calculations the sector in which the interference signal cannot be suppressed using the considered antenna system.Based on the diagram shown in Fig. 8, it can be seen that the arrival direction of the electromagnetic wave is determined by the formula

$$
\theta=\arcsin \frac{\lambda \psi}{\pi d}=\arcsin \left[\frac{\lambda}{\pi d} \operatorname{arcctg}\left(\frac{U_{12}}{U_{13}}\right)\right]
$$

The angular error has the following value

$$
\Delta \theta=\frac{1}{\sqrt{1-v^{2}}}\left(\frac{\partial v}{\partial U_{12}} \Delta U_{12}+\frac{\partial v}{\partial U_{13}} \Delta U_{13}+\frac{\partial v}{\partial \lambda} \Delta \lambda+\frac{\partial v}{\partial d} \Delta d\right)
$$

where $v=\left[\frac{\lambda}{\pi d} \operatorname{arctg}\left(\frac{U_{12}}{U_{13}}\right)\right], \Delta U_{12}, \Delta U_{13}, \Delta \lambda, \Delta d$ are measurement errors of the corresponding values.

Partial derivatives are inherently the influence coefficients. Their meanings are equal

$$
\frac{\partial v}{\partial U_{12}}=\frac{\lambda}{\pi d} \frac{U_{13}^{2}}{U_{12}^{2}+U_{13}^{2}} \frac{U_{12}}{U_{13}} \frac{1}{U_{12}} ; \frac{\partial v}{\partial \lambda}=v \frac{1}{\lambda} ; \frac{\partial v}{\partial U_{13}}=\frac{\lambda}{\pi d} \frac{U_{13}^{2}}{U_{12}^{2}+U_{13}^{2}} \frac{U_{12}}{U_{13}} \frac{1}{U_{13}} ; \frac{\partial v}{\partial d}=v \frac{1}{d}
$$

The error values $\Delta U_{12}$ and $\Delta U_{13}$ can be found using thegeneration method of the voltages $U_{12}$ and $U_{13}$. It follows from Fig. 8 that the voltage $U_{12}$ is the average value of the product of voltages $\dot{U}_{6}$ and $\dot{U}_{8}$ or

$$
U_{12}=K_{\mathrm{I}} \int_{0}^{T} M\left[\dot{U}_{5}(t) \dot{U}_{6}(t)-\dot{U}_{6}^{2}(t)\right] d t
$$

The voltages $\dot{U}_{5}$ and $\dot{U}_{6}$ contain at least two harmonic processes, one of which is related to the useful signal, the second is related to the interference signal. Therefore, neglecting small second-order quantities, we obtain

$$
\Delta U_{12}=K_{\mathrm{i}} M\left\{\left[\dot{U}_{5}(t) \Delta \dot{U}_{6}(t)+\dot{U}_{6}(t) \Delta \dot{U}_{5}(t)\right]-2 \dot{U}_{6}(t) \Delta \dot{U}_{6}(t)\right\} .
$$

The error $\Delta U_{13}$ has the same meaning. The difference lies only in the additional error that may arise during theformation of the signal $\dot{U}_{13}$ and is caused by the inaccuracy of setting the phase shift $-\pi / 2$ in the phase shifter. If this error is neglected, then $\Delta U_{13} \approx \Delta U_{12}$. Frequency settings can be made with high precision. Also, the error in determining the distance $d$ between the elements can be negligible. These prerequisites make it possible to significantly simplify formula (16), that is 


$$
\Delta \theta=\frac{2 \lambda}{\pi d \sqrt{1-\left[\frac{\lambda}{\pi d} \operatorname{arctg} \frac{U_{12}}{U_{13}}\right]^{2}}} \frac{U_{13}^{2}}{U_{12}^{2}+U_{13}^{2}} \frac{U_{12}}{U_{13}} \delta U_{12},
$$

where $\delta U_{12}$ is arelative error of measurement of the voltage $U_{12}$.

The value of the error $\Delta \theta$ varies depending on the ratio $d / \lambda$ and $U_{12} / U_{13}$. These dependencies are represented by graphs shown in Fig. 11-13 that are constructed using formula (17).

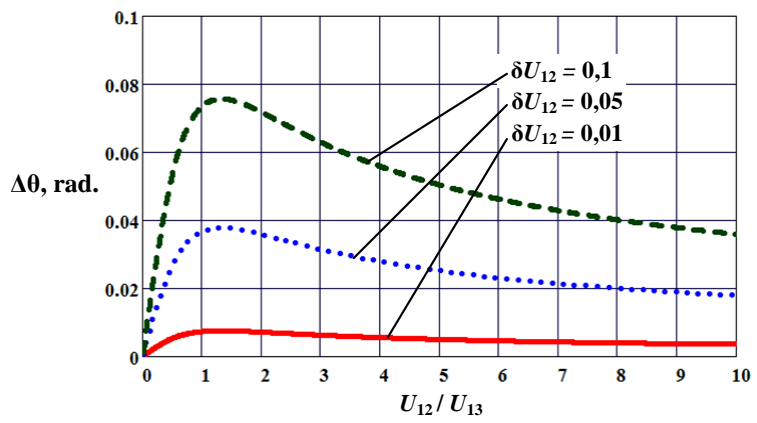

Fig.11. Dependences of the angular error in measuring the arrival direction of the interference signal on the ratio $U_{12} / U_{13}$ at the fixed value of the relative distance between the elements of the antenna array $d / \lambda=0,5$ and the relative error $\delta U_{12}$ in voltage measurement.

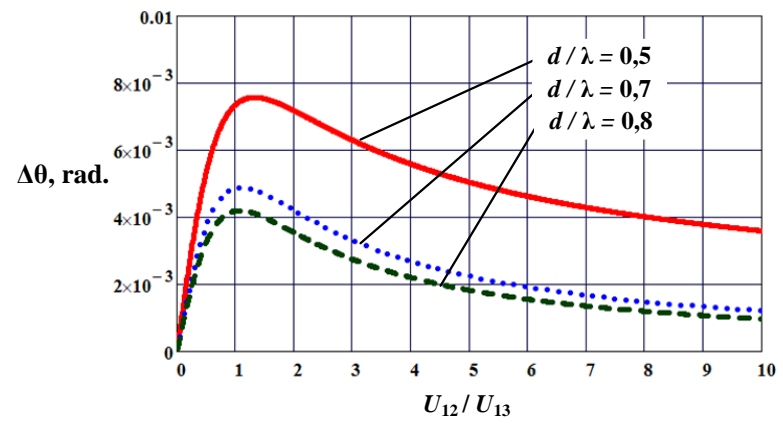

Fig.12. Dependences of the angular error in measuring the arrival direction of the interference signal on the ratio $U_{12} / U_{13}$ at the fixed values of the relative distance $d / \lambda$ between the elements of the antenna array and the relative error $\delta U_{12}=0.01$ in voltage measurement

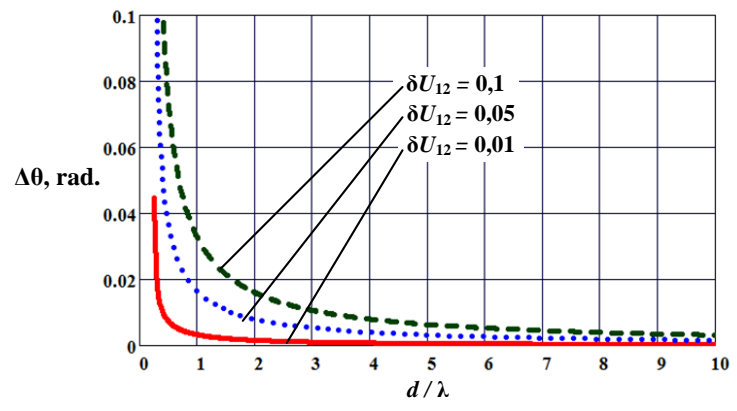

Fig.13. Dependences of the angular error in measuring the arrival direction of the interference signal on the relative distance $d / \lambda$ between the elements of the antenna array at the fixed value of the ratio $U_{12} / U_{13}=1$ and the relative error $\delta U_{12}$ in voltage measurement

As one can be seen from the graphs (Fig. 11-13), the accuracy of determining the meridional angle of arrival of the interference signal wave depends on the accuracy of constructing the antenna array elements and on the quality of the received signal processing circuit.

More complex structural diagrams of antenna systems with extended functionality for radio monitoring stations, as well as analysis of errors in measuring field parameters by these systems, are considered in the works [68-71].

There is also a need to ensure the required level of reliability and maintainability during the operation of radioelectronic equipment, which may include radio monitoring antenna systems. In the articles [72], the analysis of the operational characteristics of the electromagnetic field parameter meters with the antenna arrays and solving the 
problem of finding functional dependence for the efficiency indicator of the diagnostic procedure was carried out.

\section{Conclusion}

In this article the review of antennas and antenna systems, which are used or can be used to measure the parameters of electromagnetic waves, has been carried out. From the results of this review one can conclude that in modern radio monitoring systems monofunctional antennas are mainly used. Therefore, multiple antennas should be applied to support all radio monitoring tasks. This not only creates certain problems in the placement of antennas, but also, in the case of mobile radio monitoring systems, narrows the functionality of the radio monitoring station itself.

As follows from the design principles of antennas that can operate in difficult electromagnetic conditions, to suppress interference, it is necessary to have information about the angular position of the signal source in advance. That is, the antenna must be oriented so that its flat aperture coincides with the plane of the wavefront of the useful signal. Since such information may be unknown, which primarily relates to mobile radio monitoring terminals, it becomes necessary to provide antenna systems with the properties of direction finders. That is, the antenna system must automatically determine the angular coordinates of the radiation sources of the useful signal and interference, and also be able to shift the antenna aperture to the required spatial position.

It is known from the literature that the developers of the antennas, which are based on compensation and adaptive interference suppression methods, focus only on the development and implementation of compensation or adaptation processes, limiting themselves only to solving electromagnetic compatibility problems. The functions of direction finding, identification of radiation, measurements of radiation field parameters and assessment of the accuracy of the obtained measurement results were ignored. This area of scientific research opens up a wide field for identifying new possibilities for constructing multifunctional antennas for radio monitoring systems and creating additional incentives for their implementation into practice. Therefore, there is a need to develop methods for building antenna systems that combine the principles of adaptation to the electromagnetic environment and the functions of direction finding, radiation identification and measurement of electromagnetic field parameters with high accuracy. This combination will significantly improve the quality of mobile radio frequency monitoring procedures in the complex electromagnetic environment. This is especially actual in modern large cities.

A novel structure of two-element antenna array has been proposed for monitoring applications. From the considered operation principle of the proposed structural diagram of a this two-element adaptive antenna, it follows that the interference at the frequency of the useful signal can be effectively suppressed by such two-element antenna array, if the radiation of the interference is at a certain angle to the direction of desirable signal arrival.

It has been shown that the minimum value of the angle depends on the dynamic characteristics of the multipliers and control signal drivers. The proposed antenna system allows determining the arrival direction of the signal using a processor with the software for calculating the meridional angle. The measurement error in determining the angular position of the interference source has been estimated. This error largely depends on the accuracy of measuring the field intensity related with voltages at the antenna input.

\section{References}

[1] Murugan S., "Compact MIMO Shorted Microstrip Antenna for 5G Applications," International Journal of Wireless and Microwave Technologies (IJWMT), Vol. 11, No. 1, 2021, pp. 22-27.

[2] Howells, P.W., "Explorations in Fixed and Adaptive Resolution at GE and SURC," IEEE Trans. Antennas Propagat., Vol. 24, No. 5, 1976, pp. 575-584.

[3] Applebaum, S.P., “Adaptive Arrays,” IEEE Trans. Antennas Propagat., Vol. 24, No. 5, 1976, pp. 585-598.

[4] Gabriel, W.F., “Adaptive Arrays. An Introduction,” Proc. IEEE, Vol. 64, 1976, pp. 239- 271.

[5] Mayhan, J.T., "Some Techniques for Evaluating the Bandwidth Characteristics of Adaptive Nulling Systems," IEEE Trans. Antennas Propagat., Vol. 27, No. 3, 1979, pp. 363-373.

[6] Mayhan, J.T., Simmons, A.J., and Cummings, W.C., "Wide-Band Adaptive Antenna Nulling Using Tapped Delay Lines," IEEE Trans. Antennas Propagat., Vol. 29, No. 6, 1981, pp. 923-936.

[7] Monzingo, R.A., and Miller, T.W., Introduction to Adaptive Arrays, New York: Wiley, 1980.

[8] Compton, Jr., R.T., Adaptive Antennas, Concepts and Performance, Upper Saddle River, NJ: Prentice-Hall, 1988.

[9] Weiner, M.M., Adaptive Antennas and Receivers, Boca Raton, FL: CRC Press, 2006.

[10] Chandran, S., (ed.), Adaptive Antenna Arrays: Trends and Applications, Berlin: SpringerVerlag, 2004.

[11] Manolakis, D.G., Ingle, V.K., and Kogon, S.M., Statistical and Adaptive Signal Processing: Spectral Estimation, Signal Modeling, Adaptive Filtering, and Array Processing, Norwood, MA: Artech House, 2005.

[12] Nitzberg, R., Adaptive Signal Processing for Radar, Norwood, MA: Artech House, 1992.

[13] Farina, A., Antenna-Based Signal Processing Techniques for Radar Systems, Norwood, MA: Artech House, 1992.

[14] Hudson, J.E., Adaptive Array Principles, New York: Peter Peregrinus, 1981.

[15] Skolnik, M.I., Introduction to Radar Systems, 3rd ed., New York: McGraw-Hill, 2001.

[16] Skolnik, M.I., (ed.), Radar Handbook, 2nd ed., New York: McGraw-Hill, 1990, pp. 12.1- 13.27.

[17] Mailloux, R.J., Phased Array Antenna Handbook, Norwood, MA: Artech House, 1994.

[18] Hansen, R.C., Phased Array Antennas, New York: Wiley, 1998. 
[19] Hansen, R.C., (ed.), Significant Phased Array Antenna Papers, Dedham, MA: Artech House, 1973.

[20] Oliner, A.A., and Knittel, G.H., (eds.), Phased Array Antennas, Dedham, MA: Artech House, 1972.

[21] Amitay, N., Galindo, V. and Wu, C.P., Theory and Analysis of Phased Array Antennas, New York: Wiley, 1972.

[22] Mayhan, J.T., "Nulling Limitations for a Multiple-Beam Antenna," IEEE Trans. Antennas Propagat., Vol. AP-24, No. 6, 1976, pp. 769-779.

[23] Mayhan, J.T., “Adaptive Nulling with Multiple-Beam Antennas,” IEEE Trans. Antennas Propagat., Vol. 26, No. 2, 1978, pp. 267-273.

[24] Johnson, R.C., and Jasik, H., Antenna Engineering Handbook, 2nd ed., New York: McGraw-Hill, 1984, Ch. 22, pp. 1-20.

[25] Ayodele, S. Oluwole, and Viranjay, M. Srivastava, "Design of Smart Antenna by Circular Pin-Fed Linearly Polarized Patch Antenna," International Journal of Wireless and Microwave Technologies (IJWMT), Vol. 6, No.3, 2016, pp.40-49.

[26] Kiruthika, R., and Shanmuganantham, T., "Design and Measurement of Novel Shaped Microstrip Antenna with DGS for Radar Applications," International Journal of Wireless and Microwave Technologies (IJWMT), Vol. 8, No. 3, 2018, pp. 33-41.

[27] Jay Guo, Y., Advances in Mobile Radio Access Networks, Dedham, MA: Artech House, 2004.

[28] Gross, F.B., Smart Antennas for Wireless Communications, New York: McGrawHill, 2005.

[29] Mailloux, Robert J., Phased Array Antenna Handbook, Dedham, MA: Artech House, 2005.

[30] Rogstad, D., Mileant, A., and Pham, T., Antenna Arraying Techniques in the Deep Space Network, New York: Wiley, 2003.

[31] Meinel, H., "Commercial applications of millimeterwaves history, present status, and future trends," IEEE Trans. Microwave Theory Techniques, Vol. 43, No. 7, 1995, pp. 1639-1653.

[32] Agbinya, J.I, et al., Advances in Broadband Communication and Networks, River Publishers, 2008.

[33] Telatar, E., "Capacity of multi-antenna Gaussian channels," European Transactions on Telecommunication., Vol. 10, No. 6, 1999, pp. 585-595.

[34] Peel, C.B., Hochwald, B.M., and Swindlehurst, A.L., "A vector-perturbation technique for near-capacity multiantenna multiuser communication-Part I: Channel inversion and regularization," IEEE Transactionon Communication, Vol. 53, No. 1, 2005, pp. 195-202.

[35] Wiesel, Ami, Eldar, Yonina C., and ShlomoShamai, "Zero-forcing precoding and generalized inverses," IEEE Transaction Signal Process., Vol. 56, No. 9, 2008, pp. 4409-4418.

[36] Hayssam Dahrouj, and Wei Yu, "Coordinated beamforming for the multicell multi-antenna wireless system," IEEE Trans. Wireless Commun., Vol. 9, No. 5, 2010, pp. 1748-1759.

[37] Qingjiang Shi, Meisam Razaviyayn, Zhi-Quan Luo, and Chen He, "An iteratively weighted MMSE approach to distributed sum-utility maximization for a MIMO interfering broadcast channel," IEEE Transactions Signal Process., Vol. 59, No. 9, 2011, pp. 4331-4340.

[38] Doan, C.H., Emami, S., Sobel, D.A., Niknejad, A.M., and Brodersen, R.W., "Design considerations for 60 GHz CMOS radios," IEEE Commun. Mag., Vol. 42, No. 12, 2004, pp. 132-140.

[39] Tuan-Do-Hong and Russer, P., "Signal processing for wideband smart antenna array applications," IEEE Microwave Mag., Vol. 5, No.1, 2004, pp. 57-67.

[40] Xiaojing Huang, Jay Guo, Y., and Bunton, John D., "A hybrid adaptive antenna array," Transactions on Wireless Communications, Vol. 9, No. 5, 2010, pp. 1770 - 1779.

[41] Goffer, A.P., Kam, M., and Herczfeld, P.R., "Design of phased arrays in terms of random subarrays," IEEE Trans. Antennas Propagation, Vol. 42, No. 6, 1994, pp. 820-826.

[42] Haupt, R.L., "Optimized weighting of uniform subarrays of unequal sizes," IEEE Trans. Antennas Propagation, Vol. 55, No. 4, 2007, pp.1207- 1210 .

[43] Mailloux, R.J., "Subarray technology for large scanning arrays," In Second European Conference Antennas Propagation (EuCAP2007), 2007, pp.1-6.

[44] Abbaspour-Tamijani, A., and Sarabandi, K., "An affordable millimeterwave beam-steerable antenna using interleaved planar subarrays," IEEE Trans. Antennas Propagation, Vol. 51, No. 9, 2003, pp. 2193-2202.

[45] Hang Li, Thomas Q. Wang, Xiaojing Huang, "Joint Adaptive AoA and Polarization Estimation Using Hybrid Dual-Polarized Antenna Arrays," IEEE Access, Vol. 7, 2019, pp. 76353-76366.

[46] Glushko, D.N., and Yanovsky, F.J., "Analysis of differential Doppler velocity for remote sensing of clouds and precipitation with dual-polarization S-band radar," International Journal of Microwave and Wireless Technologies (IJMWT), Vol. 2, Special Issue 3-4, 2010, pp. 391-398.

[47] Yanovskij, F.I., and Panits, V.A., "Application of an antenna with controlled polarization for the detection of hailandicing zones" Izvestiya VUZ: Radioelektronika, Vol. 39, Issue 10, 1996, pp. 32-42.

[48] Zhang, J.A., Huang, X., Dyadyuk, V., and Guo, Y. J., "Massive hybrid antenna array for millimeter-wave cellular communications," IEEE Wireless Commun., Vol. 22, No. 1, 2015, pp. 79-87.

[49] Andrew Zhang, J., Wei Ni, Peng Cheng, and Yin Lu, "Angle-of-arrival estimation using different phase shifts across subarrays in localized hybrid arrays," IEEE Commun. Lett., Vol. 20, No. 11, 2016, pp. 2205-2208.

[50] Kai Wu, Wei Ni, Tao Su, Ren Ping Liu, and Y. JayGuo, "Robust unambiguous estimation of angle-of-arrival in hybrid array with localized analog subarrays," IEEE Trans. Wireless Commun., Vol. 17, No. 5, 2018, pp. 2987-3002.

[51] Hang Li, Tomas Q. Wang, Xiaojing Huang, and Y. Jay Guo, "Adaptive AoA and polarization estimation for receiving polarized mmWave signals," IEEE Wireless Commun. Lett.,Vol. 8, No. 2, 2019, pp. 540-543.

[52] Slobodyanyuk, P.V., Blagodarniy, V.G., and Stupak V.S., Handbook of radio monitoring, Nizhyn: TOV "Vydavnytstvo "Aspekt-Polihraf", 2008 (in Ukranian).

[53] Handbook on Spectrum Monitoring. The International Geneva: Telecommunication Union, 2002.

[54] Youcef Hacene, and Xie Shuguo, "Study of a Novel Ultra-Wideband Monopole Antenna for EMC Measurement Applications," In Proc. of the 6th Asia-Pacific Conference on Environmental Electromagnetics (CEEM), 2012, pp. 393-395.

[55] Jian Yang, and Ahmed A. Kishk, "Two New Types of Compact Ultra-wideband Antennas for EMC Measurements," In Proc. of International Symposium on Electromagnetic Compatibility (EMC Europe 2014), 2014, pp. 5-8. 
[56] Guan-Yu Chen, Jwo-Shiun Sun, Sen-Yi Huang, Chuang-Jen Huang, Kuo-Liang Wu, YD Chen, and Cheng-Hung Lin, “The Tapered TEM Horn Antenna Design for EMC and Radiation Measurement," In Proc. of 7th International Symposium on Antennas, Propagation \& EM Theory(ISAPE), 2006, pp. 1-4.

[57] Zhang Hou, Li Weimin, Wang Jian, and Ma Laixuan, "A Novel Small Sized Biconical Broadband Antenna for EMC Test Application," In Proc. of Circuits and Systems International Conference on Testing and Diagnosis (CAS-ICTD), 2009, pp. 1-4.

[58] Shcherbyna, O., and Zadorozhniy, R., "The log-periodic dipole array antenna for monitoring," In Proc. of 14th International Conference on Advanced Trends in Radioelecrtronics, Telecommunications and Computer Engineering (TCSET), 2018, pp. 583-586.

[59] Ilnytsky, L.Ya.,Sibruk, L.V., Slobodyanyuk, P.V. and Blagodarniy, V.G., Antennas of telecommunication and monitoring systems, Kyiv: Ukrainian State Center of Radio Frequencies, 2012 (in Ukranian).

[60] Melvin M. Weiner, Adaptive Antennas and Receivers, Boca Raton: CRC Press, 2006.

[61] Alan J. Fenn, Adaptive Antennas, and Phased Arrays for Radar and Communications, Boston: Artech House, 2008.

[62] John Litva, and Titus Lo, Digital Beamforming in Wireless Communications, Norwood: Artech House, 1996.

[63] Balanis, C.A., and Ioannides, P.I., Introduction to smart antennas, San Rafael, United States: Morgan and Claypool Publishers, 2007.

[64] Monzingo, R.A., Haupt, R.L., Miller, T.W., Introduction to adaptive arrays: 2 edition, New York, United States: Scitech Publishing, 2011.

[65] Haupt, R. L., Antenna Arrays: A Computational Approach, New York: Wiley, 2010.

[66] Milligan, T. A., Modern antenna design, New Jersey: John Wiley \& Sons, 2005.

[67] Ilnitskiy, L.Ya., Sibruk, L.V., Shcherbyna, O.A.,and Turabi Osama,"Errors in determining the direction of arrival of the interference signal with a two-element adaptive antenna," Problems of informatization and management, Vol. 4, No. 28, 2009, pp. 66-71 (in Russian).

[68] Ilnitskiy, L.Ya., and Shcherbyna, O.A., "Antenna system for measuring electromagnetic field parameters," Radioelectronics and Communications Systems, Vol. 57, No. 1, 2014, pp. 50-60.

[69] Ilnitskiy, L.Ya.,Shcherbyna, O.A., and Mykhalchuk I.I., "Metrological characteristics of antenna system for measuring electromagnetic field parameters," Radioelectronics and Communications Systems, Vol. 59, No 3, 2016, pp. 141-149.

[70] Ilnitskiy, L.Ya., Shcherbyna, O.A., and Mykhalchuk I.I., "Antenna System of Radiomonitoring with Advanced Features," In Proceedings of 4th IEEE International Conference Methods and Systems of Navigation and Motion Control, 2016, pp. 1-4.

[71] Shcherbyna, O.A., Ilnitskiy, L.Ya., Mykhalchuk I.I., and Kozhokhina O.V., "Accuracy characteristics of radiomonitoring antennas," Radio Electronics, Computer Science, Control, Vol. 64, No 3, 2020, pp. 42-55.

[72] Yashanov, I., Shcherbyna, O., Zaliskyi, M., Kozhokhina, O., Odarchenko, R., and Tereshchenko, L., "Diagnostics Program Efficiency Analysis for Antenna System," // In Proceedings of 15th IEEE International Conference Advanced Trends in Radioelectronics, Telecommunication and Computer Engineering (TCSET), 2020, pp. 198-202.

\section{Authors' Profiles}

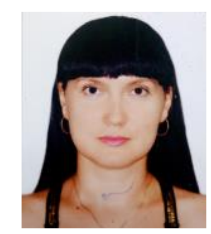

Dr Olga Shcherbyna is an associate professor at the National Aviation University. Her qualifications are as mentioned Ph.D. (Radio Engineering \& Television Systems), M.Tech. (Radio Engineering), B.Tech. (Radio Engineering). She has 15 years of teaching experience and her areas of interest include Antenna Systems, Microwave Devices, Electromagnetic Compatibility. A total number of research publications is 73 .

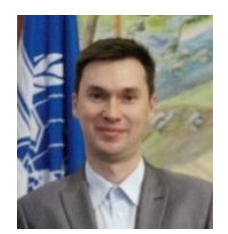

Dr Maksym Zaliskyi is an associate professor at the National Aviation University. His qualifications are as mentioned D.Sc.(Equipment Operation \& Data Processing), PhD(Equipment Operation \& Data Processing), M.Tech. (Radio Engineering), B.Tech. (Radio Engineering). He has 14 years of teaching experience and his areas of interest include Data Processing, Equipment Operation, Reliability Analysis. A total number of research publications is 130 .

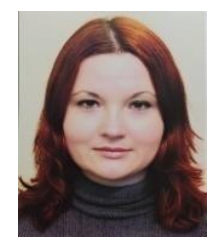

Dr Olena Kozhokhina is an associate professor at the National Aviation University. Her qualifications are as PhD (Navigation and Traffic Control), M.Tech. (Aircraft Equipment), B.Tech. (Aviation and Astronautics). She has 14 years of teaching experience and her areas of interest include Human Factors, Equipment Operation, Reliability Analysis. A total number of research publications is 72 . 
Prof. Felix Yanovsky, DSc, PhD, IEEE Life Fellow is currently a professor of electronics, robotics, monitoring and IoT technologies department at the National Aviation University, Kyiv, Ukraine. His research activity is in the field of radar, remote sensing, Doppler polarimetry, multi-parametric and adaptive signal processing and math modeling. He is the author or co-author of more than 500 scientific papers, 11 books, and 40 invention patents; served as the Chairman, TPC Chair or Section organizer of numerous IEEE and EuMA International Conferences. $\mathrm{He}$ is the State Prize Winner of Ukraine in the field of Science and Technology.

How to cite this paper: Olga Shcherbyna, Maksym Zaliskyi, Olena Kozhokhina, Felix Yanovsky, "Prospect for Using Low-Element Adaptive Antenna Systems for Radio Monitoring Stations", International Journal of Computer Network and Information Security(IJCNIS), Vol.13, No.5, pp.1-17, 2021. DOI: 10.5815/ijcnis.2021.05.01 